\title{
AUTOMORPHISM GROUPS OF SPACES OF MINIMAL RATIONAL CURVES ON FANO MANIFOLDS OF PICARD NUMBER 1
}

\author{
JUN-MUK HWANG AND NGAIMING MOK
}

\begin{abstract}
Let $X$ be a Fano manifold of Picard number 1 and $M$ an irreducible component of the space of minimal rational curves on $X$. It is a natural problem to understand the extent to which the geometry of $X$ is captured by the geometry of $M$. In this vein we raise the question as to whether the canonical map $\operatorname{Aut}_{o}(X) \rightarrow \operatorname{Aut}_{o}(M)$ is an isomorphism. After providing a number of examples showing that this may fail in general, we show that the map is indeed an isomorphism under the additional assumption that the subvariety of $M$ consisting of members passing through a general point $x \in X$ is irreducible and of dimension $\geq 2$.
\end{abstract}

\section{Introduction}

Let $X$ be a Fano manifold of Picard number 1 embedded in a projective space $\mathbf{P}_{N}$. Suppose that $X$ is covered by lines and let $M$ be an irreducible component of the Hilbert scheme of lines covering $X$. For $x \in X$, let us denote by $M^{x} \subset M$ the subscheme consisting of members of $M$ passing through $x$. By the condition that $X$ has Picard number 1 , the cardinality $\sharp\left(M^{x}\right)$ of the underlying variety $M^{x}$ is strictly bigger than 1 for general $x \in X$ (e.g. by [HM1, Proposition 13]). $\operatorname{Aut}_{o}(X)$, the identity component of the automorphism group of $X$, acts naturally on $M$. This gives a natural homomorphism $\operatorname{Aut}_{o}(X) \rightarrow \operatorname{Aut}_{o}(M)$. From $\sharp\left(M^{x}\right) \geq 2$, this homomorphism is injective. It is very natural to ask when these two groups are isomorphic. Let us examine a few examples.

Example 1. When $X=\mathbf{P}_{n}=\mathbf{P}_{N}, M$ is just the Grassmannian $\operatorname{Gr}(2, n+1)$ of 2-planes in $\mathbf{C}^{n+1}$ and $M^{x}$ is isomorphic to $\mathbf{P}_{n-1}$ for each $x \in X$. It is well known that $\operatorname{Aut}_{o}\left(\mathbf{P}_{n}\right)=\operatorname{Aut}_{o}(\operatorname{Gr}(2, n+1))$.

Received April 9, 2002. The first author was supported by Grant No. 98-0701-01-5-L from the KOSEF. The second author was supported by a CERG of the Research Grants Council of Hong Kong. 
As a matter of fact, the same holds for the family of lines lying on a Grassmannian under the Plücker embedding, and also for many rational homogeneous spaces. However, this is not always true:

Example 2. For the three-dimensional smooth hyperquadric $X$, the space $M$ of lines on $X$ is isomorphic to $\mathbf{P}_{3}$ and $M^{x} \cong \mathbf{P}_{1}$. Thus $\operatorname{Aut}_{o}(X)=$ $P S O(5, \mathbf{C})$, while $\operatorname{Aut}_{o}(M)=P S L(4, \mathbf{C})$. Another homogeneous example is the following. The exceptional simple Lie group $G_{2}$ has two simple roots, a long root $\sigma_{l}$ and a short root $\sigma_{s}$. There are three parabolic subgroups up to conjugacy: $P_{l}$ associated to $\sigma_{l}, P_{s}$ associated to $\sigma_{s}$, and the Borel subgroup $B$. Let $X=G_{2} / P_{l}, Q=G_{2} / P_{s}$, and $F=G_{2} / B$. There are natural projections $\mu: F \rightarrow X$ and $\rho: F \rightarrow Q$ induced by the inclusions $B \subset P_{l}, B \subset P_{s}$. Both $\mu$ and $\rho$ are $\mathbf{P}_{1}$-bundles. It turns out that fibers of $\rho$ are sent to curves on $X$ which are lines under the minimal projective embedding of $X$ and in fact all lines on $X$ arise this way. Thus $Q$ is naturally isomorphic to the Hilbert scheme $M$ of lines on $X$. It is well known that $Q$ is isomorphic to the fivedimensional smooth hyperquadric. Since $\mu$ is a $\mathbf{P}_{1}$-bundle, $M^{x} \cong \mathbf{P}_{1}$ for each $x \in X$. In this case, $\operatorname{Aut}_{o}(X)=G_{2}$, $\operatorname{while}_{\operatorname{Aut}_{o}}(M)=P S O(7, \mathbf{C})$.

Example 3. Let $\lambda_{1}, \ldots, \lambda_{2 g+2} \in \mathbf{C}$ be $2 g+2$ distinct points and $B$ the hyperelliptic curve of genus $g$ branched at these points. Consider two hyperquadrics

$$
\begin{aligned}
& \mathbf{Q}_{1}=\left(\sum_{i=1}^{2 g+2} X_{i}^{2}=0\right) \\
& \mathbf{Q}_{2}=\left(\sum_{i=1}^{2 g+2} \lambda_{i} X_{i}^{2}=0\right)
\end{aligned}
$$

in $\mathbf{P}_{2 g+1}$. Desale and Ramanan ([DR]) have shown that the variety of $(g-2)$ dimensional planes in the intersection $\mathbf{Q}_{1} \cap \mathbf{Q}_{2}$ is isomorphic to $\mathcal{S} U_{B}(2 ; 1)$, the moduli space of stable bundles of rank 2 with a fixed determinant of degree 1 on $B$, and the variety of $(g-1)$-dimensional planes in $\mathbf{Q}_{1} \cap \mathbf{Q}_{2}$ is isomorphic to the Jacobian $J a c_{B}$. When $g=3$, the 5-dimensional Fano variety $X=\mathbf{Q}_{1} \cap \mathbf{Q}_{2}$ has $M \cong \mathcal{S} U_{B}(2 ; 1)$. For general $x \in X, M^{x}$ is isomorphic to the complete intersection of two quadrics in $\mathbf{P}_{4}$. In this case, $\operatorname{Aut}_{o}(X) \cong\{1\} \cong \operatorname{Aut}_{o}(M)$ by [NR, Theorem 1 (a)]. On the other hand, when $g=2, M \cong J a c_{B}$ for the 3 -dimensional Fano variety $X=\mathbf{Q}_{1} \cap \mathbf{Q}_{2}$. For a general point $x \in X, M^{x}$ is isomorphic to the intersection of two conics in $\mathbf{P}_{2}$, namely, $M^{x}$ consists of 4

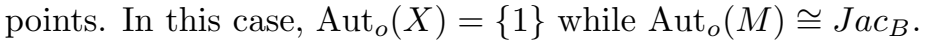

Example 4. Let $X$ be the smooth Fano threefold defined as the codimension-3 linear section of the Grassmannian $\operatorname{Gr}(2,5)$ of 2-planes in $\mathbf{C}^{5}$ under the Plücker embedding. By $\mathrm{MU}, X$ is a smooth equivariant compactification of 
$S L(2, \mathbf{C})$ modulo the octahedral group and $\operatorname{Aut}_{o}(X) \cong P S L(2, \mathbf{C})$. A line corresponds to the orbit of the Cartan subgroup of $S L(2, \mathbf{C})$. The space $M$ of all lines lying on $X$ is isomorphic to $\mathbf{P}_{2}$ ([IS, III.1.6]); thus $\operatorname{Aut}_{o}(M) \cong$ $\operatorname{Aut}_{o}\left(\mathbf{P}_{2}\right)=P S L(3, \mathbf{C}) . M^{x}$ is finite.

We list the above examples with the dimension of $M^{x}$ at general $x \in X$ :

\begin{tabular}{|c||c|c|c|c|c|}
\hline $\mathrm{X}$ & Ex. 1 & Ex. 2 & Ex. 3, g=3 & Ex. 3, g=2 & Ex. 4 \\
\hline \hline $\operatorname{dim}\left(M^{x}\right)$ & $n-1$ & 1 & 2 & 0 & 0 \\
\hline Aut $_{o}(X)=\operatorname{Aut}_{o}(M) ?$ & Yes. & No. & Yes. & No. & No. \\
\hline
\end{tabular}

Note that $\operatorname{Aut}_{o}(X)=\operatorname{Aut}_{o}(M)$ when $\operatorname{dim}\left(M^{x}\right) \geq 2$ in the above table. Our main result says that this is true in general, with one additional assumption, the irreducibility of $M^{x}$. In fact, we will prove this for general Fano manifolds of Picard number 1 where minimal rational curves play the role of lines.

Theorem 1. Let $X$ be a Fano manifold of Picard number 1, $M$ an irreducible component of the space of minimal rational curves on $X$ as defined in Section 2, and let $M^{x}$ be the subset consisting of members of $M$ passing through a general point $x \in X$. If $M^{x}$ is irreducible and $\operatorname{dim}\left(M^{x}\right) \geq 2$, then $\operatorname{Aut}_{o}(X)=\operatorname{Aut}_{o}(M)$.

The definition of $M$ given in Section 2 follows that of [Ko]. There is a natural morphism $M \rightarrow \operatorname{Chow}(X)$ which is the normalization of its image $M^{\text {Chow }}$. The natural inclusions

$$
\operatorname{Aut}_{o}(X) \subset \operatorname{Aut}_{o}\left(M^{\text {Chow }}\right) \subset \operatorname{Aut}_{o}(M)
$$

show that the statement of Theorem 1 holds when the space of minimal rational curves is understood as a subvariety of Chow $(X)$. Let $M^{\text {Hilb }}$ be the corresponding subvariety in the Hilbert scheme. Then the natural morphism $M^{\text {Hilb }} \rightarrow M^{\text {Chow }}$ is birational. Denoting the normalization of $M^{\text {Hilb }}$ by $\widehat{M^{H i l b}}$, we have the following inclusions:

$$
\operatorname{Aut}_{o}(X) \subset \operatorname{Aut}_{o}\left(M^{H i l b}\right) \subset \operatorname{Aut}_{o}\left(\widehat{M^{H i l b}}\right) \subset \operatorname{Aut}_{o}(M) .
$$

Thus the statement of Theorem 1 holds when the space of minimal rational curves is understood in the sense of Hilbert scheme.

Note that $\operatorname{dim}\left(M^{x}\right)+2$ is the anti-canonical degree of the minimal rational curves. So the condition $\operatorname{dim}\left(M^{x}\right) \geq 2$ is satisfied if the index of $X$ is $\geq 4$. When $X \subset \mathbf{P}_{N}$ and members of $M$ are lines of $\mathbf{P}_{N}$, the tangent morphism $\tau_{x}: M^{x} \rightarrow \mathbf{P} T_{x}(X)$ assigning a line through $x$ to its tangent vector at $x$, is an embedding because a line is determined by its tangent vector. Since $M^{x}$ is smooth for general $x \in X$ (cf. Section 2), we see that $M^{x}$ is irreducible if 
$\operatorname{dim}\left(M^{x}\right)>\frac{1}{2} \operatorname{dim}\left(\mathbf{P} T_{x}(X)\right)$. Thus

Corollary 1. Let $X \subset \mathbf{P}_{N}$ be a Fano manifold of Picard number 1 covered by lines and $M$ an irreducible component of the Hilbert scheme of lines covering $X$. If $K_{X}^{-1}=\mathcal{O}(k)$ with $k \geq \frac{\operatorname{dim}(X)}{2}+2$, then $\operatorname{Aut}_{o}(X)=\operatorname{Aut}_{o}(M)$.

For a smooth complete intersection $X$ of multi-degree $\left(d_{1}, \ldots, d_{k}\right)$ in $\mathbf{P}_{N}$, $X$ is covered by lines if $N-\sum_{i=1}^{k} d_{i} \geq 1$ (e.g. [Ko, V. 4.10]). In this case, the image of the tangent morphism $\tau_{x}\left(M^{x}\right) \subset \mathbf{P} T_{x}(X)$ is a complete intersection of dimension $N-1-\sum_{i=1}^{k} d_{i}$ for a general point $x \in X$. In fact, the equations defining $\mathcal{C}_{x}$ arise from [SR, I.3.1, equation (3)] when $X$ is a hypersurface. The equations defining $\mathcal{C}_{x}$ for a smooth complete intersection are obtained by repeating the calculation for the hypersurface case. Thus

Corollary 2. Let $X$ be a smooth complete intersection of multi-degree $\left(d_{1}, \ldots, d_{k}\right)$ in $\mathbf{P}_{N}$. If $N-\sum_{i=1}^{k} d_{i} \geq 3$, then the identity component of the automorphism group of the Hilbert scheme of lines on $X$ is isomorphic to that of $X$.

Theorem 1 is a direct consequence of the following local Torelli type result. The statement below is somewhat sketchy. See Section 4 for the precise statement.

Theorem 2. Let $\pi: \mathcal{X} \rightarrow \Delta$ be a regular family of Fano manifolds of Picard number 1 over the unit disc. Assume that the collection of a component $M_{t}$ of the space of minimal rational curves on the fibers $X_{t}$ of $\pi$ for $t \in \Delta$ form a flat family $\psi: \mathcal{M} \rightarrow \Delta$ and for each $t \in \Delta$ and a general point $x_{t} \in X_{t}$, $M_{t}^{x_{t}}$ is irreducible and of dimension $\geq 2$. If there exists a family of biregular morphisms $g_{t}: M_{0} \rightarrow M_{t}$ with $g_{0}=I d$, then $\left\{g_{t}\right\}$ is induced by a family of biregular morphisms $f_{t}: X_{0} \rightarrow X_{t}$ with $f_{0}=I d$.

The proof of Theorem 2 is obtained by a study of special subvarietes in $M_{t}$ corresponding to points of $X_{t}$. The condition $\operatorname{dim}\left(M_{t}^{x}\right) \geq 2$ is needed in the Kodaira vanishing of the cohomology of a natural line bundle. One technical difficulty arises from the possibility of singularity for minimal rational curves. To handle this difficulty, we need Kebekus' result ( $\mathrm{Ke}]$ ) about singularity of minimal rational curves through a general point of $X$. For the case when $X$ is covered by lines or conics under a projective embedding, this problem does not arise and our proof can be considerably shortened.

From the examples we examined, the condition $\operatorname{dim}\left(M^{x}\right) \geq 2$ is a necessary condition. But the irreducibility of $M^{x}$ is satisfied in our examples as long as $\operatorname{dim}\left(M^{x}\right) \geq 1$. We are not aware of any example $X$ and $M$ for which $\operatorname{dim}\left(M^{x}\right) \geq 1$ for general $x \in X$ but $M^{x}$ is not irreducible. In this sense, the irreducibility condition on $M^{x}$ may eventually be removable in the statement of Theorem 1 and Theorem 2, although it is essential in the proof presented below. 


\section{Spaces of minimal rational curves}

Let $X$ be a Fano manifold of Picard number 1. Choose a component $\mathcal{H}$ of the space $\operatorname{Hom}_{b i r}\left(\mathbf{P}_{1}, X\right)$ parametrizing morphisms from $\mathbf{P}_{1}$ to $X$ which are birational over their images, such that

(i) the images of elements of $\mathcal{H}$ cover a dense open subset of $X$;

(ii) for an element $h \in \mathcal{H}$, the degree of $h^{*} K_{X}^{-1}$ is minimal among all possible choices of $\mathcal{H}$ satisfying (i).

From (i), a general element of $\mathcal{H}$ is free, namely, the locally free sheaf $h^{*} T(X)$ on $\mathbf{P}_{1}$ is semi-positive. Let $\mathcal{H}^{\text {free }}$ be the open subset of $\mathcal{H}$ corresponding to free rational curves. The scheme $\mathcal{H}$ is smooth at each point $[h] \in \mathcal{H}^{\text {free }}$ because $H^{1}\left(\mathbf{P}_{1}, h^{*} T(X)\right)=0$ and the tangent space of $\mathcal{H}$ at $[h]$ is naturally isomorphic to $H^{0}\left(\mathbf{P}_{1}, h^{*} T(X)\right)$. The group $\operatorname{Aut}\left(\mathbf{P}_{1}\right)$ acts on $\mathcal{H}$. Under this action, $\mathcal{H}$ has a structure of $\operatorname{Aut}\left(\mathbf{P}_{1}\right)$-principal bundle over an irreducible quasi-projective variety $M$ which is a component of $\operatorname{RatCurves}^{n}(X)$, the normalization of the subvarieties in $\operatorname{Chow}(X)$ corresponding to rational curves of $X$ ( $\mathrm{Ko}, \mathrm{II} .2 .15])$. We call $M$ a minimal dominating component of RatCurves ${ }^{n}(X)$ and members of $M$ minimal rational curves. The subset $M^{\text {free }} \subset M$ corresponding to $\mathcal{H}^{\text {free }}$ is Zariski open and smooth. The tangent space of $M$ at a point corresponding to $[h] \in \mathcal{H}^{\text {free }}$ is naturally isomorphic to the quotient

$$
H^{0}\left(\mathbf{P}_{1}, h^{*} T(X)\right) / H^{0}\left(\mathbf{P}_{1}, T\left(\mathbf{P}_{1}\right)\right)
$$

where $T\left(\mathbf{P}_{1}\right)$ is regarded as a subsheaf of $h^{*} T(X)$ by the differential of $h$.

Let $o \in \mathbf{P}_{1}$ be a marked base point and $\operatorname{Aut}\left(\mathbf{P}_{1}, o\right)$ the subgroup of Aut $\left(\mathbf{P}_{1}\right)$ consisting of automorphisms fixing $o$. For a general point $x$ of $X$ let $\mathcal{H}^{x}$ be the subscheme of $\mathcal{H}$ corresponding to elements of $\mathcal{H}$ sending $o$ to $x$. Then $\mathcal{H}^{x} \subset \mathcal{H}^{\text {free }}$. Since $h^{*} T(X)$ is a semi-positive bundle on $\mathbf{P}_{1}$, $H^{1}\left(\mathbf{P}_{1}, h^{*} T(X) \otimes \mathbf{m}_{o}\right)=0$ for any $[h] \in \mathcal{H}^{x}$, where $\mathbf{m}_{o}$ is the maximal ideal at $o$. Thus $\mathcal{H}^{x}$ is smooth and the tangent space at a point $[h] \in \mathcal{H}^{x}$ is naturally isomorphic to $H^{0}\left(\mathbf{P}_{1}, h^{*} T(X) \otimes \mathbf{m}_{o}\right)$.

By the natural action of $\operatorname{Aut}\left(\mathbf{P}_{1}, o\right)$ on $\mathcal{H}^{x}, \mathcal{H}^{x}$ has a structure of Aut $\left(\mathbf{P}_{1}, o\right)$-principal bundle over a smooth quasi-projective variety $M^{x}$ with finitely many components ([KO, II.2.16, II.3.11.5]). The minimality of the degree of $h^{*} K_{X}^{-1}$ in the choice of $\mathcal{H}$ implies that $M^{x}$ is a projective variety. The tangent space of $M^{x}$ at a point corresponding to $[h] \in \mathcal{H}^{x}$ is naturally isomorphic to the quotient

$$
H^{0}\left(\mathbf{P}_{1}, h^{*} T(X) \otimes \mathbf{m}_{o}\right) / H^{0}\left(\mathbf{P}_{1}, T\left(\mathbf{P}_{1}\right) \otimes \mathbf{m}_{o}\right) .
$$

Although $\mathcal{H}^{x}$ is a submanifold of $\mathcal{H}$, the induced morphism $\eta: M^{x} \rightarrow$ $M^{\text {free }} \subset M$ is not necessarily an embedding. Let us see at what point $\eta$ is not injective. Two elements $h_{1}, h_{2} \in \mathcal{H}^{x}$ are sent to the same point in $M$ if 
and only if they have the same image in $X$. If they are different as elements of $M^{x}, h_{1}(o)=h_{2}(o)=x$ must correspond to a multiple point of the image $C:=h_{1}\left(\mathbf{P}_{1}\right)=h_{2}\left(\mathbf{P}_{2}\right)$ and an analytic neighborhood of $o \in \mathbf{P}_{1}$ is sent to different branches of $C$ at $x$ by $h_{1}$ and $h_{2}$.

Proposition 1. The morphism $\eta: M^{x} \rightarrow M^{\text {free }}$ is an immersion for a general point $x \in X$.

Proof. Since $\mathcal{H}^{x} \subset \mathcal{H}^{\text {free }}$ is an embedding, $\eta$ is an immersion at $[h] \in M^{x}$ if the $\operatorname{Aut}\left(\mathbf{P}_{1}\right)$-orbit of $[h]$ slices $\mathcal{H}^{x}$ along the $\operatorname{Aut}\left(\mathbf{P}_{1}, o\right)$-orbit of $[h]$ in a transversal way. This is equivalent to

$$
H^{0}\left(\mathbf{P}_{1}, T\left(\mathbf{P}_{1}\right)\right) \cap H^{0}\left(\mathbf{P}_{1}, h^{*} T(X) \otimes \mathbf{m}_{o}\right)=H^{0}\left(\mathbf{P}_{1}, T\left(\mathbf{P}_{1}\right) \otimes \mathbf{m}_{o}\right) .
$$

This is precisely the case if $h: \mathbf{P}_{1} \rightarrow X$ is an immersion at $o$. Thus Proposition 1 follows from the following result of S. Kebekus.

Proposition 2 ( $\mathrm{Ke}$ Theorem 3.3 and 3.4]). If $x \in X$ is general, any $[h] \in \mathcal{H}^{x}$ is an immersion at o, i.e., $h_{*} T_{o}\left(\mathbf{P}_{1}\right) \neq 0$. Furthermore, the tangent morphism $\tau_{x}: M^{x} \rightarrow \mathbf{P} T_{x}(X)$ defined by $\tau_{x}([h])=h_{*} T_{o}\left(\mathbf{P}_{1}\right)$ is a finite morphism over its image.

The restriction of the universal $\mathbf{P}_{1}$-bundle $\operatorname{Univ}^{r c}(X) \rightarrow \operatorname{RatCurves}^{n}(X)$ to $M$ gives the universal $\mathbf{P}_{1}$-bundle $\rho: \mathcal{U} \rightarrow M$ with a natural morphism $\mu: \mathcal{U} \rightarrow X$. Furthermore, there exists a natural morphism $U: \mathbf{P}_{1} \times \mathcal{H} \rightarrow \mathcal{U}$ so that $\rho \circ U=u \circ p_{2}$ where $p_{2}: \mathbf{P}_{1} \times \mathcal{H} \rightarrow \mathcal{H}$ is the projection and $u: \mathcal{H} \rightarrow M$ is the quotient by $\operatorname{Aut}\left(\mathbf{P}_{1}\right)$ ([Ko, II.2.15]):

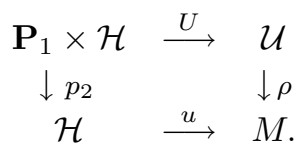

Let $j: \mathcal{H}^{x} \rightarrow \mathcal{U}$ be the restriction of $U(o, *): \mathcal{H} \rightarrow \mathcal{U}$. This descends to $\iota: M^{x} \rightarrow \mathcal{U}$ which is injective. The composition $\rho \circ \iota: M^{x} \rightarrow M$ is exactly the immersion $\eta$ in Proposition 1. It follows that $\iota$ is an embedding and we can identify $M^{x}$ with the submanifold $\iota\left(M^{x}\right)$ in $\mathcal{U}$. Then the restriction of $\rho$ : $\mathcal{U} \rightarrow M$ to $\iota\left(M^{x}\right)$ coincides with $\eta$ in Proposition 1. Under this identification, $M^{x}=\mu^{-1}(x)$. In the following, by $T(\mathcal{U})$ and $T(M)$, we denote the tangent bundles of the smooth parts of $\mathcal{U}$ and $M$, respectively.

Proposition 3. Let $L$ be the line subbundle of $T(\mathcal{U})$ defined by the relative tangent vectors of the $\mathbf{P}_{1}$-bundle $\rho: \mathcal{U} \rightarrow M$. Then $L^{-1}$ restricted to $M^{x}$ is ample.

Proof. By the definition of the tangent morphism $\tau_{x}: M^{x} \rightarrow \mathbf{P} T_{x}(X)$ in Proposition 2, $L$ restricted to $M^{x}$ is equivalent to the pull-back of the tautological line bundle $\mathcal{O}(-1)$ on $\mathbf{P} T_{x}(X)$ by $\tau_{x}$. Since $\tau_{x}$ is finite by Proposition $2, L^{-1}$ is ample on $M^{x}$. 
Proposition 4. On $M^{x}$, we have the following short exact sequence

$$
0 \longrightarrow L \longrightarrow \mathcal{O}^{n} \longrightarrow \eta^{*} T(M) / T\left(M^{x}\right) \longrightarrow 0
$$

where $T\left(M^{x}\right)$ is regarded as a subbundle of $\rho^{*} T(M)$ by the immersion $\eta$ : $M^{x} \rightarrow M$.

Proof. By the definition of $L$, we have the following exact sequence on $\mathcal{U}$ :

$$
0 \longrightarrow L \longrightarrow T(\mathcal{U}) \longrightarrow \rho^{*} T(M) \longrightarrow 0 .
$$

Let $N_{M^{x}} \subset \mathcal{U}$ be the normal bundle of $M^{x}$ in $\mathcal{U}$. Restricting the above sequence to $M^{x}$ and quotienting out by $T\left(M^{x}\right)$, we get

$$
0 \longrightarrow L \longrightarrow N_{M^{x} \subset \mathcal{U}} \longrightarrow \eta^{*} T(M) / T\left(M^{x}\right) \longrightarrow 0 .
$$

Since $N_{M^{x}} \subset \mathcal{U}$ is a trivial bundle, we get the desired sequence.

\section{Spaces of immersed subvarieties}

Let $Y$ be an irreducible quasi-projective variety. An irreducible projective subvariety $Z \subset Y$ is called an immersed subvariety if $Z$ lies in the smooth locus of $Y$, its normalization $\hat{Z}$ is smooth and the normalization morphism $\nu: \hat{Z} \rightarrow Z$ is unramified. The set of immersed subvarieties of $Y$ form a subscheme $\operatorname{Imm}_{Y}$ of the Hilbert scheme of $Y$. Let $\theta: \mathcal{W} \rightarrow \operatorname{Imm}_{Y}$ be the universal family. Given a subscheme $V \subset \operatorname{Imm}_{Y}$, the restriction of $\theta$ to $V$ will be denoted by $\theta_{V}: \mathcal{W}_{V} \rightarrow V$.

Take the underlying reduced varieties of all the schemes involved and regard $\mathcal{W}_{V}$ and $V$ as varieties. Let $\varphi: \hat{\mathcal{W}}_{V} \rightarrow \mathcal{W}_{V}$ be the normalization of $\mathcal{W}_{V}$. Then a general fiber of $\theta_{V} \circ \varphi$ gives the normalization of the corresponding fiber of $\theta_{V}: \mathcal{W}_{V} \rightarrow V$ by Seidenberg's theorem (e.g. [BS, Theorem 1.7.1]). In particular, there exists a nonempty open subvariety $V^{\prime}$ of $V$ so that we have a simultaneous normalization of fibers of $\theta_{V^{\prime}}: \mathcal{W}_{V^{\prime}} \rightarrow V^{\prime}$. Thus there exists a natural stratification of $\operatorname{Imm}_{Y}$ into countably many subschemes so that over each stratum the universal family can be simultaneously normalized in the above sense. We will call this $\mathbf{S N}$-stratification (SN standing for "simultaneous normalization").

For an immersed subvariety $\nu: \hat{Z} \rightarrow Z \subset Y$, the quotient $\nu^{*} T(Y) / T(\hat{Z})$ is a vector bundle on $\hat{Z}$, which will be denoted by $N_{\hat{Z}}$. Here $T(Y)$ means the tangent bundle of the smooth locus of $Y$.

Proposition 5. For an immersed subvariety $Z \subset Y$ of a quasi-projective variety $Y$, let $\hat{Z}$ be its normalization and $\mathcal{S}$ the $S N$-stratum of $\operatorname{Imm}_{Y}$ containing the point $[Z]$. Then $\operatorname{dim}(\mathcal{S}) \leq \operatorname{dim} H^{0}\left(\hat{Z}, N_{\hat{Z}}\right)$. 
Proof. Let $\hat{\mathcal{W}}_{\mathcal{S}} \rightarrow \mathcal{S}$ be the simultaneous normalizations of the universal family over $\mathcal{S}$. To prove the claimed inequality, we may assume that $[Z]$ is a general point of $\mathcal{S}$, by the upper-semi-continuity of $\operatorname{dim} H^{0}\left(\hat{Z}, N_{\hat{Z}}\right)$ as $Z$ varies.

By the natural morphism $\mu: \mathcal{W} \rightarrow Y$ associated to the universal family, $\hat{\mathcal{W}}_{\mathcal{S}} \rightarrow \mathcal{S}$ can be viewed as a deformation of the morphism $\nu: \hat{Z} \rightarrow Y$ in the sense of [Ho]. We have the Kodaira-Spencer map defined in section 1 of [Ho]

$$
\kappa: T_{[Z]}(\mathcal{S}) \rightarrow H^{0}\left(\hat{Z}, N_{\hat{Z}}\right),
$$

which must be injective because $[Z]$ is general in $\mathcal{S}$.

Let us apply the above discussions to the case $Y=M$, a minimal dominating component of RatCurves ${ }^{n}(X)$ for a Fano manifold $X$ of Picard number 1. Assume that $M^{x}$ is irreducible for general $x \in X$. Let $\rho: \mathcal{U} \rightarrow M, \mu: \mathcal{U} \rightarrow X$ be the universal family morphisms. Restricting to a Zariski dense open subset $X^{\prime} \subset X$, we may assume that $\mu^{\prime}:\left.\mathcal{U}\right|_{X^{\prime}} \rightarrow X^{\prime}$ is a smooth morphism with connected fibers whose fibers over $x \in X^{\prime}$ correspond to $M^{x}$. By shrinking $X^{\prime}$ if necessary, we may assume that $\rho$ gives an immersion of each fiber of $\mu^{\prime}$ into $M^{\text {free }}$ by Proposition 1. Thus we have, by a simultaneous normalization, a morphism $\sigma: X^{\prime} \rightarrow \operatorname{Imm}_{M}$ defined by

$$
\left.\sigma(x):=\quad \text { the image of } M^{x} \text { in } M\right] .
$$

By shrinking $X^{\prime}$ further, we can assume that $\sigma\left(X^{\prime}\right)$ lies in a single SN-stratum $\mathcal{S}$ of $\operatorname{Imm}_{M}$ and $\mu^{\prime}:\left.\mathcal{U}\right|_{X^{\prime}} \rightarrow X^{\prime}$ is the pull-back of the simultaneous normalization of the universal family over $\mathcal{S}$.

Proposition 6. If $\operatorname{dim}\left(M^{x}\right) \geq 1$, the morphism $\sigma: X^{\prime} \rightarrow \mathcal{S}$ defined above is generically injective.

Proof. Suppose $\sigma\left(x_{1}\right)=\sigma\left(x_{2}\right)$ for general $x_{1} \neq x_{2}$ in $X^{\prime}$. This means that $M^{x_{1}}=M^{x_{2}}$. Thus we have a positive dimensional family of rational curves belonging to $M$ in $X$ passing through $x_{1}$ and $x_{2}$. Such a family must degenerate to a reducible curve passing through $x_{1}$ and $x_{2}$ by bend-and-break ([Ko, II.5]). This is a contradiction to the minimal degree condition (ii) in the choice of $\mathcal{H}$.

Proposition 7. Suppose $\operatorname{dim}\left(M^{x}\right) \geq 2$. Then $\sigma: X^{\prime} \rightarrow \mathcal{S}$ is birational.

Proof. By Proposition 6, it suffices to show that $\operatorname{dim}(\mathcal{S}) \leq n:=\operatorname{dim}(X)$. By Proposition 5, it is enough to show that for a general fiber $F$ of $\mu^{\prime}:\left.\mathcal{U}\right|_{X^{\prime}} \rightarrow$ $X^{\prime}, \operatorname{dim} H^{0}\left(F, N_{F}\right)=n . F$ is an irreducible component of $M^{x}$ for a general $x \in X$. From Proposition 4,

$$
0 \longrightarrow H^{0}(F, L) \longrightarrow H^{0}\left(F, \mathcal{O}^{n}\right) \longrightarrow H^{0}\left(F, N_{F}\right) \longrightarrow H^{1}(F, L) \longrightarrow \cdots
$$

But from the assumption that $\operatorname{dim}(F) \geq 2$, by Kodaira's Vanishing Theorem for negative line bundles we get $H^{1}(F, L)=0=H^{0}(F, L)$ because $L^{-1}$ 
is ample on $M^{x}$ by Proposition 3. It follows that $h^{0}\left(F, N_{F}\right)=h^{0}\left(F, \mathcal{O}^{n}\right)$ $=n$.

For a general $h \in M$, let $\mathcal{S}^{h} \subset \mathcal{S}$ be the subscheme consisting of members of $\mathcal{S}$ passing through $h$. From Proposition 7, the following is evident.

Proposition 8. For a general member $h: \mathbf{P}_{1} \rightarrow X$ of $M$,

$$
\mathcal{S}^{h}=\text { closure of } \bigcup_{\text {general }}\left[M^{x}\right]
$$

where $\left[M^{x}\right]$ denotes the point of $\mathcal{S}$ corresponding to the immersed image of $M^{x}$ in $M$. Moreover, under the birational map $\sigma: X \rightarrow \mathcal{S}$ of Proposition 7, $\sigma\left(h\left(\mathbf{P}_{1}\right)\right)=\mathcal{S}^{h}$ for general $h \in M$.

\section{Proof of Theorem 2}

We can state Theorem 1 in a precise form as follows.

Theorem 1. Let $X$ be a Fano manifold of Picard number 1 and $M a$ minimal dominating component of RatCurves ${ }^{n}(X)$. Suppose for a general $x \in X, M^{x}$ is irreducible and of dimension $\geq 2$. Then $\operatorname{Aut}_{o}(X)=\operatorname{Aut}_{o}(M)$.

Theorem 1 is a direct consequence of the following Theorem 2, applied to the case $\mathcal{X}=X \times \Delta$ and $\mathcal{M}=M \times \Delta$.

Theorem 2. Let $\pi: \mathcal{X} \rightarrow \Delta$ be a regular family of Fano manifolds of Picard number 1 over the unit disc. Assume that there exists a component of $\operatorname{Hom}_{\text {bir }}\left(\mathbf{P}_{1}, \mathcal{X}\right)$ which gives a flat family of projective varieties $\psi: \mathcal{M} \rightarrow \Delta$ so that $M_{t}:=\psi^{-1}(t)$ is a minimal dominating component for $X_{t}:=\pi^{-1}(t)$ for each $t \in \Delta$. Suppose $M_{t}^{x_{t}}$ is irreducible of dimension $\geq 2$ for a general $x_{t} \in X_{t}$ for all $t \in \Delta$. If there exists a family of biregular morphisms $g_{t}: M_{0} \rightarrow M_{t}$ with $g_{0}=\mathrm{Id}$. Then $\left\{g_{t}\right\}$ is induced by a family of biregular morphisms $f_{t}$ : $X_{0} \rightarrow X_{t}$ with $f_{0}=$ Id.

Proof. The biregular morphism $g_{t}$ induces a biregular morphism $g_{t *}$ : $\operatorname{Imm}_{M_{0}} \rightarrow \operatorname{Imm}_{M_{t}}$ which preserves the SN-stratifications. Let $\mathcal{S}_{t}$ be the corresponding stratum of $\operatorname{Imm}_{M_{t}}$ arising from $X_{t}$ for each $t \in \Delta$. By Proposition 7 , we have the following sequence of birational maps:

$$
X_{0} \stackrel{\sigma_{0}}{\longrightarrow} \mathcal{S}_{0} \stackrel{g_{t *}}{\longrightarrow} \mathcal{S}_{t} \stackrel{\sigma_{t}^{-1}}{\longrightarrow} X_{t}
$$


Let $f_{t}: X_{0} \rightarrow X_{t}$ be the birational map which is the composition of the above, namely, $f_{t}=\sigma_{t}^{-1} \circ g_{t *} \circ \sigma_{0}$. For a general member $h: \mathbf{P}_{1} \rightarrow X$ of $M_{0}$,

$$
\begin{aligned}
f_{t}\left(h\left(\mathbf{P}_{1}\right)\right) & =\sigma_{t}^{-1}\left(g_{t *}\left(\sigma_{0}\left(h\left(\mathbf{P}_{1}\right)\right)\right)\right) \\
& =\sigma_{t}^{-1}\left(g_{t *}\left(\mathcal{S}_{0}^{h}\right)\right) \text { by Proposition } 8 \\
& =\sigma_{t}^{-1}\left(\mathcal{S}_{t}^{g_{t}(h)}\right) \\
& =\sigma_{t}^{-1}\left(\sigma_{t}\left(g_{t}(h)\left(\mathbf{P}_{1}\right)\right)\right) \text { by Proposition } 8 \\
& =g_{t}(h)\left(\mathbf{P}_{1}\right)
\end{aligned}
$$

where the image under a rational map means the strict image. Thus the birational map $f_{t}: X_{0} \rightarrow X_{t}$ sends members of $M_{0}$ to members of $M_{t}$ and vice versa. It follows that $f_{t}$ can be extended to a biregular morphism from $X_{0}$ to $X_{t}$ by the following proposition, which is equivalent to Proposition 4.4 in [HM2]. We reproduce the proof for the readers' convenience.

Proposition 9. Let $X_{1}, X_{2}$ be two Fano manifolds of Picard number 1. Let $M_{1}$ resp. $M_{2}$ be a minimal rational component on $X_{1}$ resp. $X_{2}$. Let $\Phi: X_{1} \rightarrow$ $X_{2}$ be a birational map sending general members of $M_{1}$ to general members of $M_{2}$ and vice versa. Then $\Phi$ can be extended to a biregular morphism.

Proof. We denote by $B \subset X$ the subvariety on which $\Phi$ fails to be a local biholomorphism.

First assume that $B$ is of codimension $\geq 2$. Since $X_{1}$ and $X_{2}$ are Fano we may choose $k$ large enough so that both $K_{X_{1}}^{-k}$ and $K_{X_{2}}^{-k}$ are very ample. Let $s$ be a pluri-anticanonical section on $X_{2}$ in $\Gamma\left(X_{2}, K_{X_{2}}^{-k}\right)$. Then $\Phi^{*} s$ is a well-defined pluri-anticanonical section on $X_{1}-B$. It extends across $B$ under the assumption that $B$ is of codimension $\geq 2$. It follows that $\Phi$ induces a linear monomorphism $\phi: \Gamma\left(X_{2}, K_{X_{2}}^{-k}\right) \rightarrow \Gamma\left(X_{1}, K_{X_{1}}^{-k}\right)$ and hence a linear isomorphism $\phi^{*}: \Gamma\left(X_{1}, K_{X_{1}}^{-k}\right)^{*} \rightarrow \Gamma\left(X_{2}, K_{X_{2}}^{-k}\right)^{*}$ by taking adjoints. Identifying $X_{1}$ resp. $X_{2}$ as a complex submanifold of $\mathbf{P} \Gamma\left(X_{1}, K_{X_{1}}^{-k}\right)^{*}$ resp. $\mathbf{P} \Gamma\left(X_{2}, K_{X_{2}}^{-k}\right)^{*}, \Phi$ is nothing other than the restriction of the projectivization $\left[\phi^{*}\right]: \mathbf{P}\left(\Gamma\left(X_{1}, K_{X_{1}}^{-k}\right)^{*}\right) \rightarrow \mathbf{P}\left(\Gamma\left(X_{2}, K_{X_{2}}^{-k}\right)^{*}\right)$ to $X_{1}$, thus a biholomorphism.

Now suppose $B$ has an irreducible component $R$ of codimension 1 in $X$. The strict image $\Phi(R)$ has codimension $\geq 2$ in $X^{\prime}$. Since $X_{1}$ has Picard number 1, all $M_{1}$-curves intersect $R$. Thus their images under $\Phi$ will intersect $\Phi(R)$. But these images are general $M_{2}$-curves by assumption, a contradiction to [KO, II.3.7]. 


\section{References}

[BS] Beltrametti, M. and Sommese, A.: The adjunction theory of complex projective varieties. Walter de Gruyter, 1995.

[DR] Desale, U.V. and Ramanan, S.: Classification of vector bundles of rank 2 on hyperelliptic curves. Invent. Math. 38 (1976) 161-185.

[Ho] Horikawa, E.: On deformations of holomorphic maps I. J. Math. Soc. Japan 25 (1973) 372-396.

[HM1] Hwang, J.-M. and Mok, N.: Rigidity of irreducible Hermitian symmetric spaces of the compact type under Kähler deformation. Invent. Math. 131 (1998) 393-418.

[HM2] Hwang, J.-M. and Mok, N.: Cartan-Fubini type extension of holomorphic maps for Fano manifolds of Picard number 1. Journal Math. Pures Appl. 80 (2001) 563-575.

[Is] Iskovskikh, V.A.: Anticanonical models of 3-dimensional algebraic varieties. J. Soviet Math. 13 (1980) 745-814.

[Ke] Kebekus, S.: Families of singular rational curves. J. Alg. Geom. 11 (2002) 245-256.

[Ko] Kollár, J.: Rational curves on algebraic varieties. Ergebnisse der Mathematik und ihrer Grenzgebiete, 3 Folge, Band 32, Springer-Verlag, 1996.

[MU] Mukai, S. and Umemura, H.: Minimal rational threefolds. in Algebraic Geometry, Tokyo/Kyoto 1982. Lecture Notes in Math. 1016 (1983) 490-518.

[NR] Narasimhan, M.S. and Ramanan, S.: Deformations of the moduli space of vector bundles over an algebraic curve. Ann. of Math. 101 (1975) 391-417.

[SR] Semple, J. G. and Roth, L.: Introduction to algebraic geometry. Oxford University Press, 1985.

Department of Mathematics, Korea Institute for Advanced Study, 207-43 Cheongryangri-dong, Seoul 130-012, Korea

E-mail address: jmhwang@ns.kias.re.kr

Department of Mathematics, The University of Hong Kong, Pokfulam Road, HONG KONG

E-mail address: nmok@hkucc.hku.hk 